\title{
Highly Polymorphous Nicotinamide and Isonicotinamide: Solution versus Melt Crystallization
}

Noalle Fellah, Carolyn Jin Zhang, Catherine Chen, Chunhua T. Hu, Bart Kahr, Michael D. Ward, Alexander G. Shtukenberg*

Department of Chemistry and Molecular Design Institute, New York University, New York City, NY, 10003, USA

\section{Supporting Information}

\section{TABLE OF CONTENTS}

Table S1. Solubility of nicotinamide and isonicotinamide in common solvents.

Table S2. Comparison of nicotinamide polymorphs.

Table S3. Summary of solution screening for nicotinamide and isonicotinamide.

Table S4. Hydrogen bonding motifs in isonicotinamide polymorphs.

Table S5. Hydrogen bonding motifs in nicotinamide polymorphs.

Figure S1. Raman spectra of nicotinamide polymorphs.

Figure S2. Raman spectra of isonicotinamide polymorphs.

Figure S3. Raman spectra of nicotinamide melt.

Table S6. Assignments of Raman spectra for nicotinamide.

Figure S4. Growth rates of nicotinamide polymorphs at high supercooling.

Table S7. Calculated lattice and surface energies for nicotinamide polymorphs.

Table S8. Calculated lattice and surface energies for isonicotinamide polymorphs. 
Table S1. Solubility of nicotinamide (NA) and isonicotinamide (INA) in common solvents at $25^{\circ} \mathrm{C}$ (data marked with * were collected at $23^{\circ} \mathrm{C}$ ).

\begin{tabular}{|c|c|c|c|c|}
\hline \multirow[t]{2}{*}{ Solvent } & \multicolumn{2}{|c|}{ Nicotinamide } & \multicolumn{2}{|c|}{ Isonicotinamide } \\
\hline & $\begin{array}{l}\mathrm{g} / 100 \mathrm{ml} \\
\text { solvent }\end{array}$ & Reference & $\begin{array}{l}\text { g/100 ml } \\
\text { solvent }\end{array}$ & Reference \\
\hline \multirow[t]{2}{*}{ water } & 72.4 & 1 & 10.5 & 2 \\
\hline & & & $10.5^{*}$ & Our data \\
\hline \multirow[t]{2}{*}{ methanol } & 28.9 & 3 & 15.3 & 4 \\
\hline & 25 & 1 & 10.3 & 5 \\
\hline \multirow[t]{2}{*}{ ethanol } & 9.7 & 3 & 7 & 5 \\
\hline & 10.1 & 1 & 6.8 & 2 \\
\hline \multirow[t]{2}{*}{ isopropanol } & 5.4 & 3 & 4.4 & 5 \\
\hline & 5.2 & 1 & 4.3 & 2 \\
\hline \multirow[t]{2}{*}{ 1-butanol } & 5.4 & 3 & 6.4 & 2 \\
\hline & 5.7 & 1 & & \\
\hline isobutanol & 5.2 & 3 & 3.9 & 2 \\
\hline \multirow[t]{2}{*}{ ethyl acetate } & 1.4 & 3 & 0.9 & 4 \\
\hline & 1.4 & 1 & 1 & 2 \\
\hline butyl acetate & 1 & 3 & 0.71 & 2 \\
\hline acetone & 3.2 & 3 & 2.5 & 4 \\
\hline acetonitrile & 1.9 & Our data & 1.6 & 4 \\
\hline dichloromethane & $0.47^{*}$ & Our data & 0.38 & 4 \\
\hline dioxane & 4.8 & Our data & 1.8 & 5 \\
\hline nitromethane & $1.7^{*}$ & Our data & 0.3 & 5 \\
\hline chloroform & $0.78^{*}$ & Our data & $0.42 *$ & Our data \\
\hline diethyl ether & $0.113^{*}$ & Our data & $0.165^{*}$ & Our data \\
\hline hexane & 0.025 & Our data & 0.018 & Our data \\
\hline toluene & 0.044 & Our data & 0.053 & Our data \\
\hline acetic acid & $21.2^{*}$ & Our data & 10.5 & Our data \\
\hline o-xylene & $0.7 *$ & Our data & $0.21 *$ & Our data \\
\hline
\end{tabular}


Table S2. Comparison of nicotinamide polymorphs. Our polymorphs were verified with Kofler \& Kolšek based on melting temperature $\left(T_{m}\right)$ and crystal morphology. The polymorphs identified by Lu et al. were verified by comparing $T_{m}$ and PXRD patterns.

\begin{tabular}{cccccc}
\hline \multicolumn{2}{c}{ This paper } & \multicolumn{2}{c}{ Kofler \& Kolšek (1971) } & \multicolumn{2}{c}{ Lu et al. $(2020)^{\mathbf{b}}$} \\
Polymorph Name & $\boldsymbol{T}_{\boldsymbol{m}},{ }^{\mathbf{}}{ }^{\mathbf{C}} \mathbf{C}$ & Polymorph Name & $\boldsymbol{T}_{\boldsymbol{m}},{ }^{\mathbf{}}{ }^{\mathbf{C}}$ & Polymorph Name & $\boldsymbol{T}_{\boldsymbol{m}},{ }^{\mathbf{}}{ }^{\mathbf{C}}$ \\
\hline I & 129.8 & I & 130 & $\alpha$ & 129 \\
II & 115.9 & II & 117 & $\beta$ & 117 \\
III & 114.5 & III & 115 & $\gamma$ & 115 \\
IV & 112.6 & V & 112 & $\delta$ & 114 \\
V & 110.6 & IV & 114 & $\varepsilon$ & 110.5 \\
VI & - & - & - & $\zeta$ & 109.5 \\
VII & 108.7 & VI & 110 & $\eta$ & 108 \\
VIII & 104.5 & VII & 106 & $\theta$ & 105 \\
IX & - & - & - & 1 & 103 \\
\hline
\end{tabular}

aef. 6

${ }^{\text {bRef. } 7}$ 
Table S3. Summary of solution screening for INA and NA.

\begin{tabular}{|c|c|c|c|c|}
\hline \multirow[b]{2}{*}{ Solvent } & \multicolumn{2}{|c|}{ NA } & \multicolumn{2}{|c|}{ INA } \\
\hline & Evaporation & $T$ lowering & Evaporation & $T$ lowering \\
\hline benzene & I & n.d. & II & n.d. \\
\hline toluene & I & n.d. & IV & n.d. \\
\hline o-xylene & I & n.d. & II & n.d. \\
\hline hexane & I & n.d. & I, II, IV, VI & n.d. \\
\hline diethyl ether & I & n.d. & I, II, IV & n.d. \\
\hline THF & I & I & II & I \\
\hline 1,4-dioxane & I & I & II & III, IV, VI \\
\hline ethyl acetate & I & I & II, IV & I, IV \\
\hline methyl acetate & I & I & II & I \\
\hline dichloromethane & I,VII & I & I, III, IV & II, IV \\
\hline pyridine & I & I & I & II, IV \\
\hline acetone & I & I & I, II, IV & I, II \\
\hline nitrobenzene & n.d. & I & n.d. & II \\
\hline nitromethane & I & I & II, IV & I \\
\hline DMF & I & I & II & IV \\
\hline acetonitrile & I & I & I, II, IV & I, II \\
\hline DMSO & n.d. & I & n.d. & II \\
\hline chloroform & I & I & IV & I, III, IV \\
\hline acetic acid & solvate $^{\mathrm{a}}$ & n.d. & solvate $^{b}$ & n.d. \\
\hline butanol & I & I & I, II, III, IV & I, II, IV \\
\hline isopropanol & I & I & I, II, III, IV & II \\
\hline ethanol & I & I & I, III & $\mathrm{I}$ \\
\hline methanol & I & I & I & I, II \\
\hline water & I & I & monohydrate $^{c}$ & II \\
\hline formamide & I & solvate $^{\mathrm{d}}$ & II & solvate $^{\mathrm{e}}$ \\
\hline $\begin{array}{l}\text { CCDC number: } 20 \\
\text { REFCODE: JAWV } \\
\text { CCDC number: } 20 \\
\text { CCDC number: } 20 \\
\text { REFCODE: GAV }\end{array}$ & $\begin{array}{l}2164 \\
A^{8} \\
2166 \\
2165 \\
R^{9}\end{array}$ & & & \\
\hline
\end{tabular}


Table S4. Hydrogen bonding motifs in isonicotinamide polymorphs.

\begin{tabular}{|c|c|c|c|}
\hline $\begin{array}{l}\text { Polymorph } \\
\text { refcode }\end{array}$ & $\begin{array}{l}\text { Space } \\
\text { group } \\
Z^{\prime} \\
\end{array}$ & $\begin{array}{l}\text { Amid D } \\
\text { Amid N } \\
\text { Pyridine }\end{array}$ & Hydrogen bonding motif \\
\hline $\begin{array}{l}\text { INA I } \\
\text { EHOWIH01 }\end{array}$ & $P 2{ }_{1} / c$ & $\begin{array}{l}\text { Yes } \\
\text { Yes } \\
\text { No }\end{array}$ & A into isolated corrugated sheets \\
\hline $\begin{array}{l}\text { INA II } \\
\text { EHOWIH02 }\end{array}$ & $\begin{array}{l}P 2{ }_{1} / c \\
2\end{array}$ & $\begin{array}{l}\text { No } \\
\text { Yes } \\
\text { Yes }\end{array}$ & Chains of molecules connected by H-bonds into isolated flat sheets \\
\hline $\begin{array}{l}\text { INA III } \\
\text { EHOWIH03 }\end{array}$ & $\begin{array}{l}\text { Pbca } \\
1\end{array}$ & $\begin{array}{l}\text { No } \\
\text { Yes } \\
\text { Yes }\end{array}$ & Chains of molecules connected by H-bonds into isolated flat sheets \\
\hline $\begin{array}{l}\text { INA IV } \\
\text { EHOWIH04 }\end{array}$ & $\begin{array}{l}P c \\
3\end{array}$ & $\begin{array}{l}\text { No } \\
\text { Yes } \\
\text { Yes }\end{array}$ & Chains of molecules connected by H-bonds into isolated flat sheets \\
\hline $\begin{array}{l}\text { INA V } \\
\text { EHOWIH05 }\end{array}$ & $\begin{array}{l}P 2{ }_{1} / c \\
1\end{array}$ & $\begin{array}{l}\text { No } \\
\text { Yes } \\
\text { Yes }\end{array}$ & Chains of molecules connected by $\mathrm{H}$-bonds into isolated corrugated sheets \\
\hline $\begin{array}{l}\text { INA VI } \\
\text { EHOWIH06 }\end{array}$ & $\begin{array}{l}P c a 2_{1} \\
2\end{array}$ & $\begin{array}{l}\text { No } \\
\text { Yes } \\
\text { Yes }\end{array}$ & 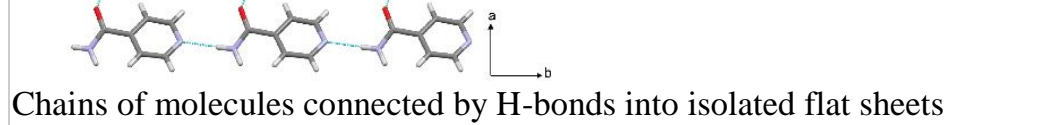 \\
\hline
\end{tabular}

Amid D = dimers $\mathrm{N}_{\text {amide }} \mathrm{H} \cdots \mathrm{O}$; Amid $\mathrm{N}=$ none dimeric $\mathrm{N}_{\text {amide }}-\mathrm{H} \ldots \mathrm{O}$; Pyridine $=\mathrm{N}_{\text {amide }}-\mathrm{H} \cdots \mathrm{N}$ pyridine 
Table S5. Hydrogen bonding motifs in nicotinamide polymorphs.

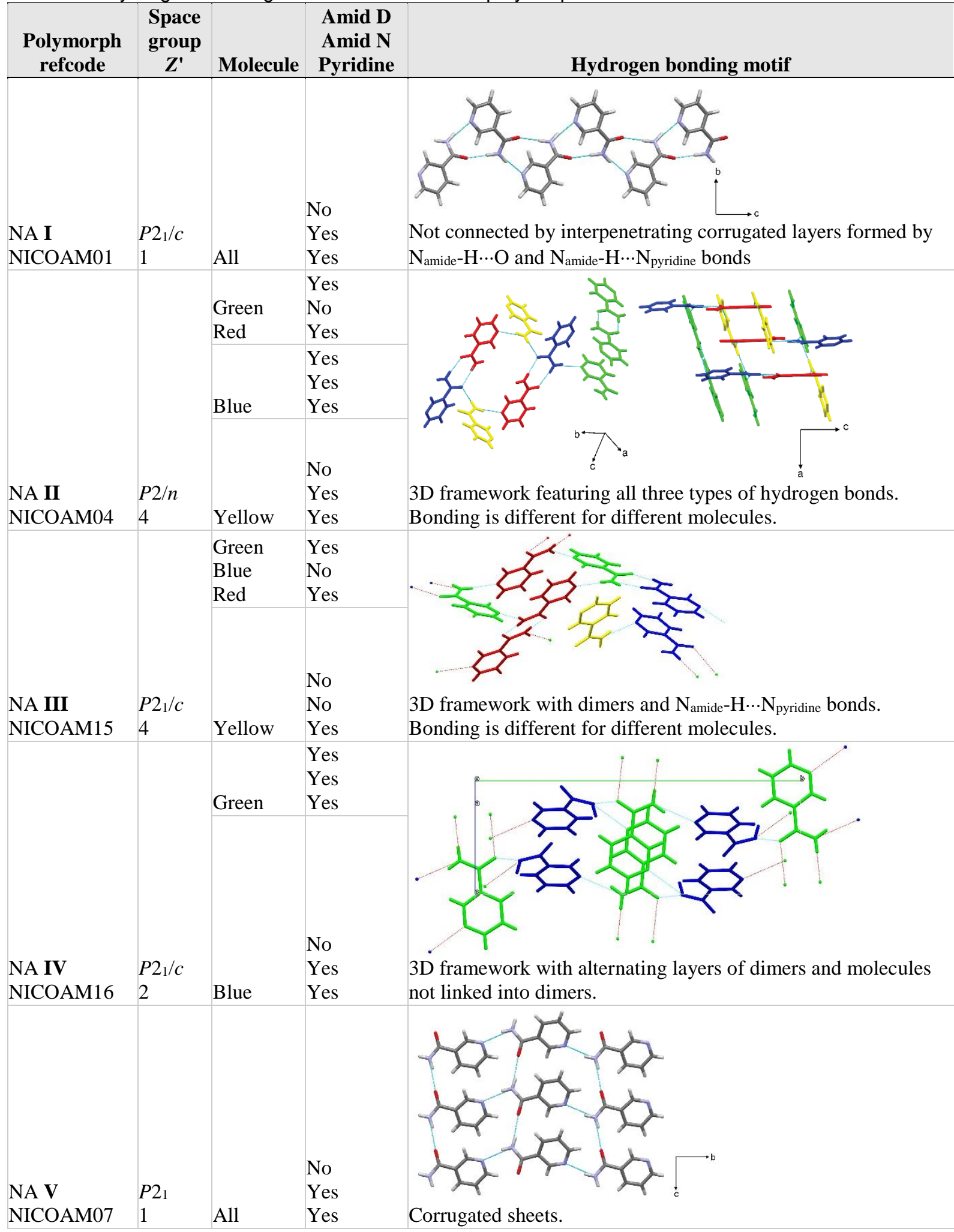


Table S5. Continued

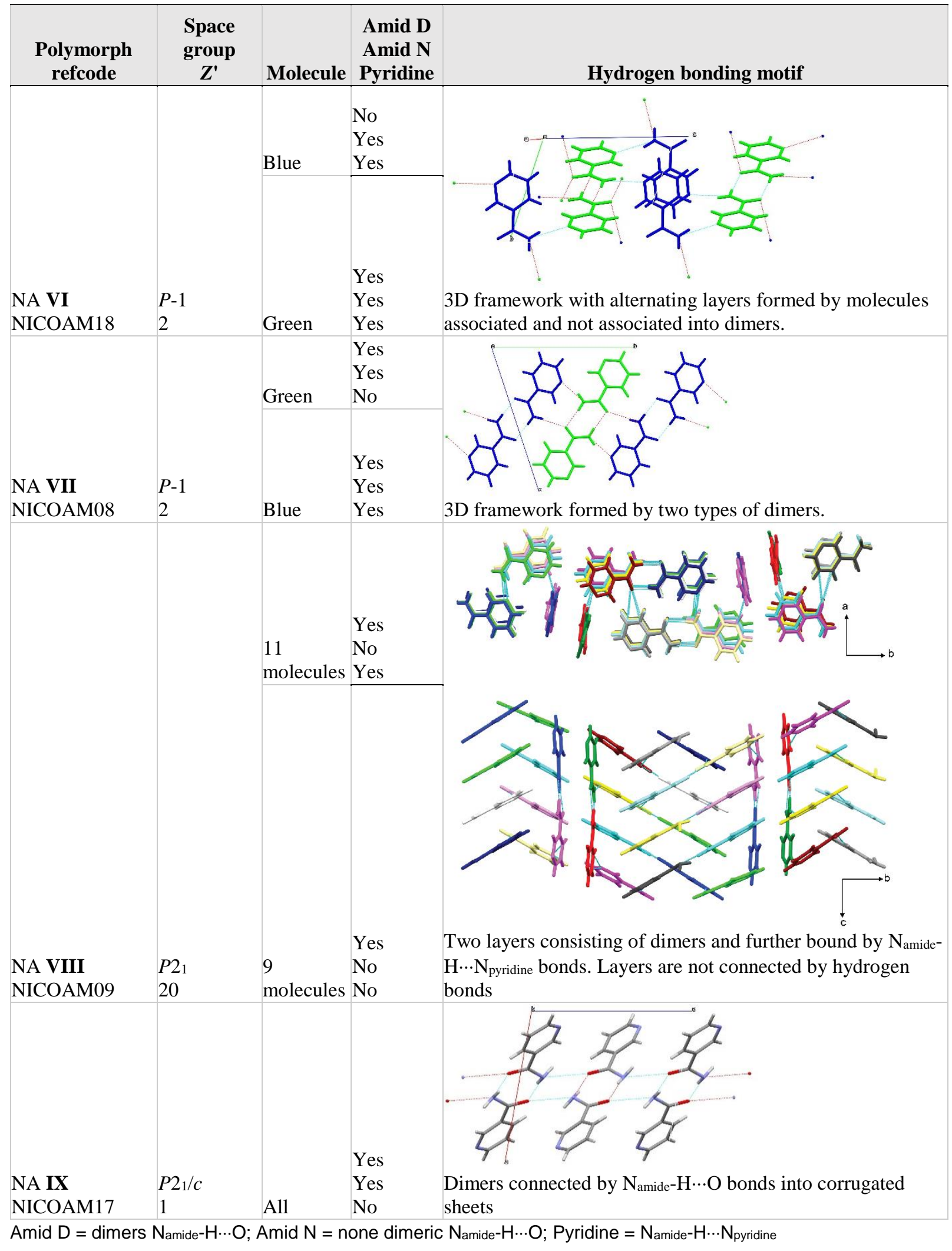



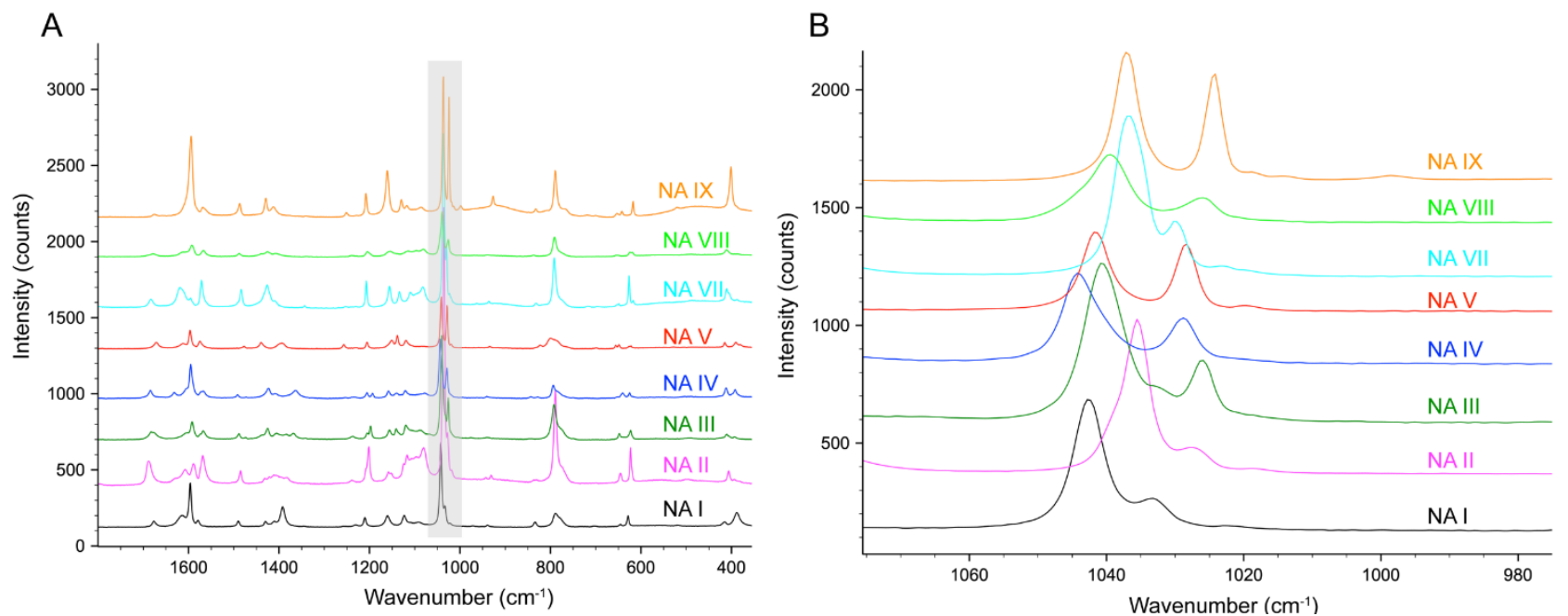

Figure S1. Raman spectra of the NA polymorphs. (B) Detail from shaded region of panel A emphasizing differences.

A

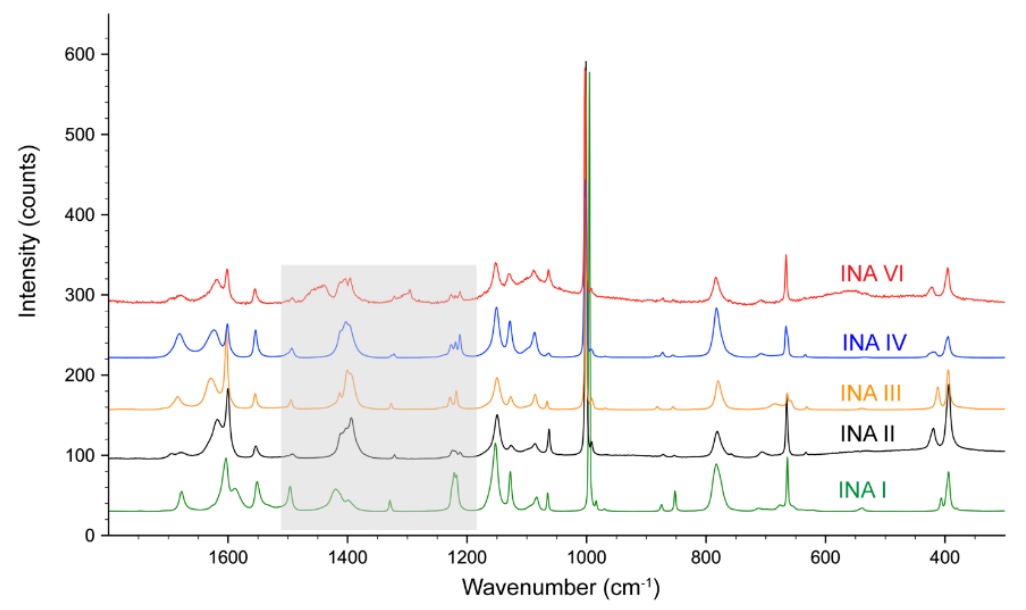

B

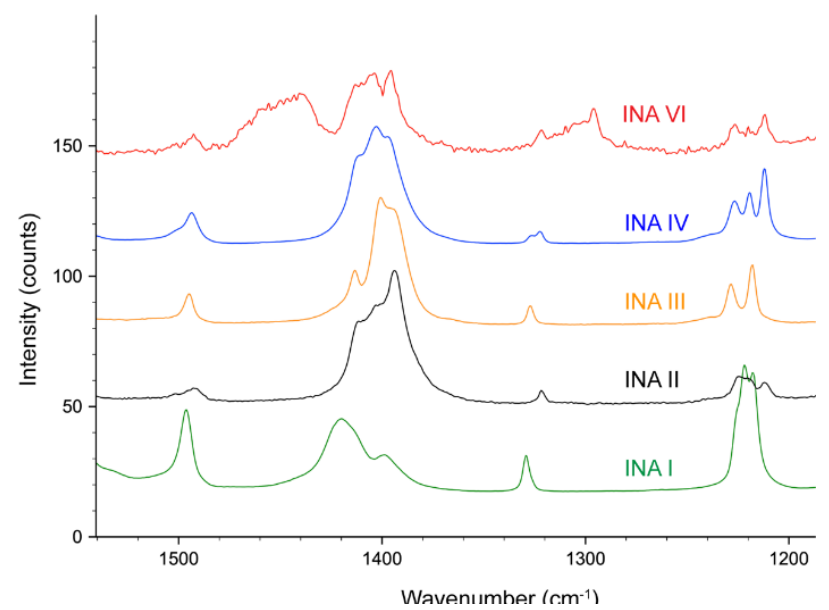

Figure S2. Raman spectra of the INA polymorphs. (B) Detail from shaded region of panel A emphasizing differences.

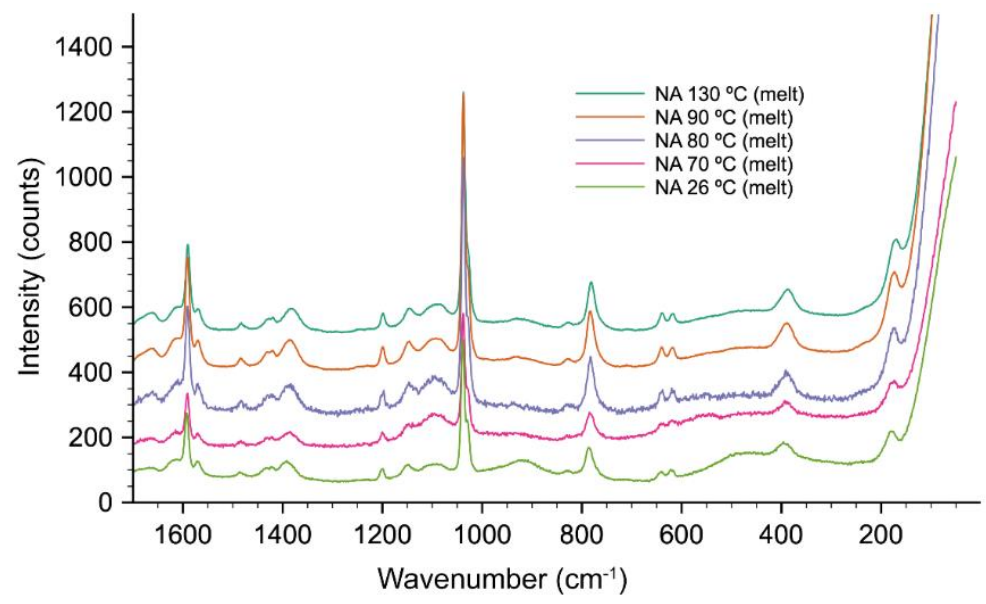

Figure S3. Raman spectra of NA melt at $26,70,80,90$, and $130 \stackrel{\circ}{C}$. 
Table S6. NA Raman assignments for NA I between 1300-1800 $\mathrm{cm}^{-1}$.

\begin{tabular}{|c|c|}
\hline Approximate Descriptiona & Wavenumber $\left(\mathrm{cm}^{-1}\right)$ \\
\hline $\mathrm{CO}$ stretch., $v(\mathrm{CO})$ & 1677 \\
\hline $\mathrm{NH}_{2}$ scissoring & 1615 \\
\hline CC stretch., $v(\mathrm{CC})$ & 1596 \\
\hline CC stretch., $v(\mathrm{CC})$ & 1578 \\
\hline $\mathrm{CH}$ ip. bend. $\delta(\mathrm{CH})$ & 1490 \\
\hline $\mathrm{CH}$ ip. bend. $\delta(\mathrm{CH})$ & 1430 \\
\hline $\mathrm{CN}_{\text {amide }}$ stretch., $v\left(\mathrm{CN}_{\text {amide }}\right)$ & 1392 \\
\hline
\end{tabular}

assignments are based on Ref. 10.

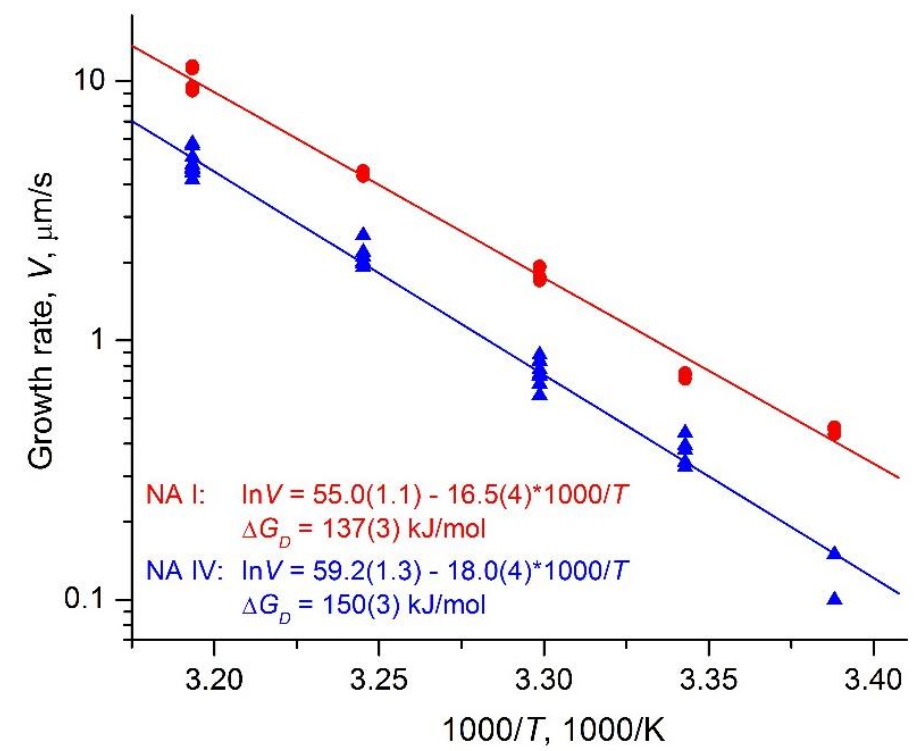

Figure S4. Growth rate, $V$, of NA I (red circles) and NA IV (blue triangles) as a function of inverse temperature $1000 / T$. The corresponding growth temperatures range from 22 to $40{ }^{\circ} \mathrm{C}$. To decrease scattering of points this plot contains only the data collected for the same sample on the same day. The lines are linear fits to determine the kinetic barrier for nucleation and growth, $\Delta G_{D}$. 
Table S7. Calculated lattice and surface energies for NA polymorphs.

\begin{tabular}{|c|c|c|c|c|c|}
\hline Polymorph & $\begin{array}{l}\text { Lattice } \\
\text { energy, } \\
\text { kcal/mol }\end{array}$ & $\bar{Z}$ & $\begin{array}{l}\text { Absolute } \\
\text { lattice } \\
\text { energy per } \\
\text { molecule, } \\
\mathrm{kJ} / \mathrm{mol}^{\text {a }}\end{array}$ & $\begin{array}{l}\text { Average } \\
\text { surface energy } \\
\text { in vacuum, } \\
\langle\gamma\rangle_{\text {vacuum, }}, \\
\mathbf{m J} / \mathbf{m}^{2 \mathbf{b}}\end{array}$ & $\begin{array}{l}\text { Average } \\
\text { crystal-melt } \\
\text { surface energy, } \\
\langle\gamma\rangle_{\text {melt }}, \mathrm{mJ} / \mathrm{m}^{2} \mathrm{c}\end{array}$ \\
\hline NA I & -105.8 & 4 & 110.7 & 180.0 & 37.4 \\
\hline NA II & -405.1 & 16 & 106.0 & 158.5 & 32.9 \\
\hline NA III & -420.6 & 16 & 110.0 & 171.7 & 35.7 \\
\hline NA IV & -215.0 & 8 & 112.5 & 189.0 & 39.3 \\
\hline NA V & -52.6 & 2 & 110.0 & 157.8 & 32.8 \\
\hline NA VI & -104.7 & 4 & 109.5 & 161.9 & 33.7 \\
\hline NA VII & -102.4 & 4 & 107.1 & 152.9 & 31.8 \\
\hline NA VIII & -1021.4 & 40 & 106.8 & 140.4 & 29.2 \\
\hline NA IX & -101.6 & 4 & 106.3 & 150.8 & 31.4 \\
\hline
\end{tabular}

aCalculated lattice energy agrees with the literature data on sublimation energy, $\Delta H_{\text {subl }}=112.1 \mathrm{~kJ} / \mathrm{mol} .{ }^{11}$

${ }^{\mathrm{b}}\langle\gamma\rangle_{\text {vacuum }}=\sum_{i} A_{i} \gamma_{i} / \sum_{i} A_{i}$, where $i$ corresponds to all $(h k l)$ faces bounding a crystal in vacuum, $A_{i}$ are areas of these faces and $\gamma_{i}$ are their surface energies.

${ }^{c}\langle\gamma\rangle_{\text {melt }}=\langle\gamma\rangle_{\text {vacuum }} \Delta H / \Delta H_{\text {subl }}$. The calculated $\langle\gamma>$ melt matches well with surface energy estimated using Turnbull expression for non-metallic crystals, $\gamma_{\text {cryst-melt }}=0.32 \Delta H / \omega^{2 / 3} N_{A}^{1 / 3}=45.7 \mathrm{~mJ} / \mathrm{m}^{2},{ }^{12}$ where $N_{A}$ is Avogadro's number.

Table S8. Calculated lattice and surface energies for INA polymorphs.

\begin{tabular}{|c|c|c|c|c|c|}
\hline Polymorph & $\begin{array}{l}\text { Lattice } \\
\text { energy, } \\
\text { kcal/mol }\end{array}$ & $Z$ & $\begin{array}{l}\text { Absolute } \\
\text { lattice } \\
\text { energy per } \\
\text { molecule, } \\
\text { kJ/mol a }\end{array}$ & $\begin{array}{l}\text { Average } \\
\text { surface energy } \\
\text { in vacuum, } \\
\langle\gamma\rangle_{\text {vacuum, }} \\
\mathrm{mJ} / \mathrm{m}^{2 \mathrm{~b}}\end{array}$ & $\begin{array}{l}\text { Average } \\
\text { crystal-melt } \\
\text { surface energy, } \\
\langle\gamma\rangle_{\text {melt, }} \mathrm{mJ} / \mathrm{m}^{2} \mathrm{c}\end{array}$ \\
\hline INA I & -105.1 & 4 & 110.0 & 180.0 & 39.6 \\
\hline INA II & -217.1 & 8 & 113.6 & 183.5 & 40.4 \\
\hline INA III & -219.3 & 8 & 114.7 & 184.2 & 40.5 \\
\hline INA IV & -163.3 & 6 & 113.8 & 180.0 & 39.6 \\
\hline INA V & -108.6 & 4 & 113.6 & 182.8 & 40.2 \\
\hline INA VI & -216.8 & 8 & 113.4 & 183.5 & 40.4 \\
\hline
\end{tabular}

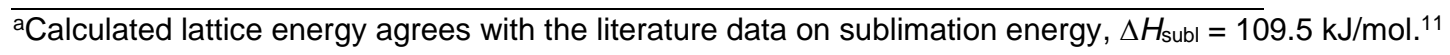

${ }^{\mathrm{b}}\langle\gamma\rangle_{\text {vacuum }}=\sum_{i} A_{i} \gamma_{i} / \sum_{i} A_{i}$, where $i$ corresponds to all ( $\left.h k l\right)$ faces bounding a crystal in vacuum, $A_{i}$ are areas of these faces and $\gamma_{i}$ are their surface energies.

${ }^{c}\langle\gamma\rangle_{\text {melt }}=\langle\gamma\rangle_{\text {vacuum }} \Delta H / \Delta H_{\text {subl }}$. The calculated $<\gamma>$ melt matches well with surface energy estimated using Turnbull expression for non-metallic crystals, $\gamma_{\text {cryst-melt }}=0.32 \Delta H / \omega^{2 / 3} N_{A}^{1 / 3}=45.9 \mathrm{~mJ} / \mathrm{m}^{2},{ }^{12}$ where $N_{A}$ is Avogadro's number. 


\section{REFERENCES}

(1) Wu, H.; Dang, L.; Wei, H. Solid-liquid phase equilibrium of nicotinamide in different pure solvents: Measurements and thermodynamic modeling. Ind. Eng. Chem. Res. 2014, 53, 1707-1711.

(2) Li, B.; Wu, Y.; Zhu, J.; Chen, K.; Wu, B.; Ji, L. Determination and correlation of solubility and mixing properties of isonicotinamide (form II) in some pure solvents. Thermochim. Acta 2016, 627-629, 55-60.

(3) Ouyang, J.; Zhang, Y.; Na, B.; Liu, Z.; Zhou, L.; Hao, H. Solubility determination of nicotinamide and its application for the cocrystallization with benzoic acid. J. Chem. Eng. Data 2018, 63, 4157-4165.

(4) Hansen, T. B.; Taris, A.; Rong, B.-G.; Grosso, M.; Qu, H. Polymorphic behavior of isonicotinamide in cooling crystallization from various solvents. J. Cryst. Growth 2016, 450, 8190.

(5) Kulkarni, S. A.; McGarrity, E. S.; Meekes, H.; ter Horst, J. H. Isonicotinamide self-association: The link between solvent and polymorph nucleation. Chem. Comm. 2012, 48, 4983-4985.

(6) Kofler, A.; Kolšek, J. Beitrag zur mikroskopischen Identifizierung organischer Stoffe nach L. Kofler. V. Microchim. Acta 1971, 59, 848-874.

(7) Li, X.; Ou, X.; Wang, B.; Rong, H.; Wang, B.; Chang, C.; Shi, B.; Yu, L.; Lu, M. Rich polymorphism in nicotinamide revealed by melt crystallization and crystal structure prediction. Commun Chem 2020, 3, 152-160.

(8) Bhogala, B. R.; Basavoju, S.; Nangia, A. Tape and layer structures in cocrystals of some di- and tricarboxylic acids with 4,4'-bipyridines and isonicotinamide. From binary to ternary cocrystals. CrystEngComm 2005, 7, 551-562.

(9) Oswald, I. D. H.; Motherwell, W. D. S.; Parsons, S. Isonicotinamide-formamide (1/1). Acta Crystallogr. E 2005, 61, o3161-o3163.

(10) Akalin, E.; Akyuz, S. Vibrational analysis of free and hydrogen bonded complexes of nicotinamide and picolinamide. Vib. Spectrosc. 2006, 42, 333-340.

(11) Zhabina, A. A.; Nagrimanov, R. N.; Emel'yanenko, V. N.; Solomonov, B. N.; Verevkin, S. P. Nicotinamides: Evaluation of thermochemical experimental properties. J. Chem. Thermodynamics 2016, 103, 69-75.

(12) Turnbull, D. Formation of crystal nuclei in liquid metals. J. Appl. Phys. 1950, 21, 1022-1028. 